\title{
The International Conference of Natural Product Biosynthesis (ICNPB, 8th US-Japan seminar on the Biosynthesis of Natural Products)
}

\author{
Takayoshi Awakawa
}

The Journal of Antibiotics (2012) 65, 587-590; doi:10.1038/ja.2012.74; published online 19 September 2012

The International Conference of Natural Product Biosynthesis, which is also called the 8th US-Japan Seminar on the Biosynthesis of Natural Products, was held at the Awaji Yumebutai in Hyogo, Japan, from 17 to 22 June 2012. The program was organized by Professor Hideaki Oikawa (Hokkaido University) and the organizing committee, which covered many important topics on biosynthesis of bioactive natural products. Ninety-two participants attended the conference composed of nine sessions. The major topics of this meeting were analyses of biosynthetic routes or enzymes to produce secondary metabolites in microbes and plants, and redesign of the metabolites by engineering the biosynthetic routes or enzymes. This report will briefly summarize some presentations that were given at the conference.

\section{KEYNOTE LECTURES}

The conference began with inspiring plenary lectures by Professor Hideaki Oikawa and Professor David Cane. Professor Oikawa presented the total biosyntheses of fungal natural products, aphidicolin and paspaline, by the heterologous expression of multiple genes in Aspergillus oryzae. ${ }^{1}$ The multiple-gene expression system expands the possibility of synthesizing various chemical structures in microbial hosts. In addition, in vitro analyses of epoxidase (Lsd18) and epoxide hydrolase (Lsd19) responsible for lasalocid biosynthesis were discussed. X-ray crystallographic analysis of Lsd19 provided detailed information of epoxide-opening cyclic ether formation. ${ }^{2}$ This study exemplified the use of crystallographic analysis as one of the most powerful tools to clarify enzymatic reaction mechanisms. In another plenary lecture, Professor David Cane (Brown University) described the regulation of the stereochemistry of intermediates by polyketide synthase (PKS), in which several ketoreductases from PKS systems, responsible for production of 6-deoxyerythronolide B, picromycin and tylactone, were shown to possess different stereospecificities to epimerize a D-2-methyl group and reduce a 3-ketoacyl group of diketide and triketide intermediates, respectively. ${ }^{3}$ To detect the stereochemically different intermediates, individual PKS domains

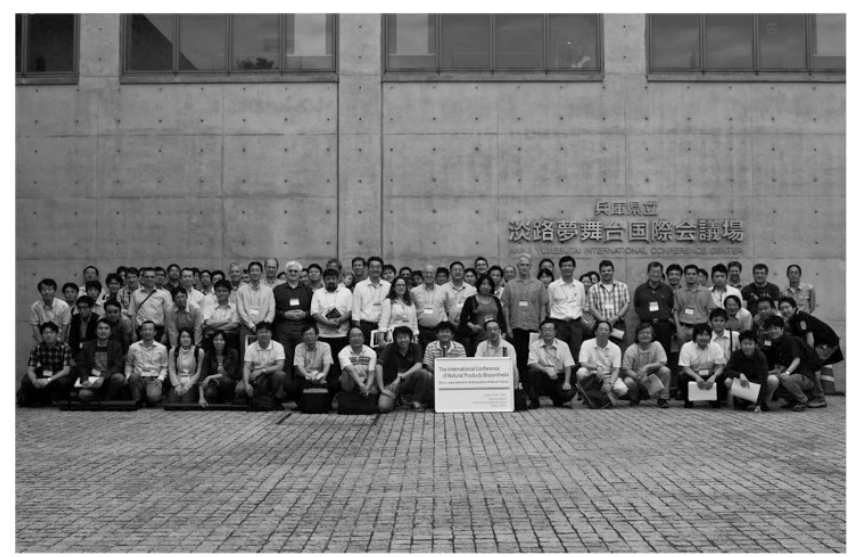

were purified separately. This study provided important insights to design chimeric PKSs capable of catalyzing stereochemically regulated reactions.

\section{POLYKETIDE AND PKS}

Session 2 (Polyketide and PKS) opened with a lecture by Professor Ben Shen (Scripps, Florida), who discussed the diversity of enediyne compounds, which are among the most potent anticancer agents, such as C-1027 and neocarzinostatin. These molecules were shown to be commonly derived from a heptaene synthesized by a single module type I PKS that catalyzes iterative chain elongation. ${ }^{4}$ Professor Ikuro Abe (University of Tokyo) presented syntheses of unnatural alkaloids using a type III PKS and nitrogen-containing substrates. ${ }^{5}$ Adding a point mutation to the enzyme increased the round of polyketide chain extension, resulting in production of a novel alkaloid. Dr Adrian Keatinge-Clay (University of Texas, Austin) reported in vitro 
biosyntheses of chiral triketide lactones using a single PKS module. A PKS module harboring several ketoreductase domains and enzymes to provide extender units and NADPH enabled this group to control the stereochemistry of the products on a preparative scale. ${ }^{6}$ Dr Nobutaka Funa (University of Shizuoka) reported that the increase of malonyl-CoA efflux in Escherichia coli was induced by reduced expression level of an enzyme responsible for fatty acid production by gene knockdown or disruption. Professor Hiroyuki Morita (Toyama University) reported the catalytic mechanism of diarylheptanoid scaffold-forming curcuminoid synthase from Oryza sativa based on its crystal structure. ${ }^{7}$ Professor Sheryl Tsai (University of California, Irvine) presented two different mechanisms to cyclize the polyketomethylene chain by the product template domain of fungal PKS (norsolorinic acid anthrone synthase) ${ }^{8}$ and the cyclase of type II PKS systems (tetracenomycin-producing system), respectively. ${ }^{9}$ Dr Kenji Arakawa (Hiroshima University) described the structural elucidation and biosynthesis of the butenolide signaling molecules that induce lankacidin and lankamycin production in Streptomyces rochei. ${ }^{10}$ Professor Jürgen Rohr (University of Kentucky) reviewed a combinatorial biosynthetic enzymology, designated in vitro reconstitution of the biosynthetic pathway and recombination of enzyme components in type II PKS systems. In this study, glycosylation, dehydration and oxidation catalyzed by enzymes of the gilvocarcin and jadomycin biosynthetic pathways were characterized. ${ }^{11}$

\section{FUNGAL PKS AND EXPRESSION}

Session 3 (Fungal PKS and expression) opened with a lecture by Professor Isao Fujii (Iwate Medical University), who reported the biosynthetic study of a type III PKS from $A$. oryzae, which produces csypyrone B1. $\left[1,2-{ }^{13} \mathrm{C}_{2}\right]$ Acetate feeding experiments showed that csypyrone B1 could be derived from succinyl-CoA and malonylCoAs. ${ }^{12}$ Dr Kenji Watanabe (University of Shizuoka) reported the formation of spirocarbon at spirotryprostatin in Saccharomyces cerevisiae by expressing $\mathrm{FAD}$-dependent monooxygenase responsible for fumiquinazoline biosynthesis. Professor Katsuya Gomi (Tohoku University) described the Cre/loxP-mediated marker recycling system. Efforts to regulate the expression of Cre in A. oryzae were discussed. Professor Russell Cox (University of Bristol) reviewed the biosynthesis of tropolones in fungi. Utilizing gene disruption, heterologous expression and in vitro assay, the tropolone biosynthetic pathway was shown to include 3-methylorcinaldehyde synthesis and three oxidation steps. ${ }^{13}$

\section{NONRIBOSOMAL PEPTIDE SYNTHETASE (NRPS), RIBOSOMAL PEPTIDE SYNTHETASE/PKS-NRPS HYBRID AND ISOPRENOIDS}

Session 4 (Nonribosomal peptide synthetase (NRPS), ribosomal peptide synthetase/PKS-NRPS hybrid and isoprenoids) opened with a lecture by Professor Yi Tang (University of California, Los Angeles), who reported an in vitro assay of NRPS to produce tryptoquialanine. The expression system in $S$. cerevisiae enabled this group to express and purify the large NRPS module. Dr Yoshimitsu Hamano (Fukui Prefectural University) described AMP esterification of L- $\beta$-lysine and amide bond-forming reaction to produce $\mathrm{L}-\beta$-lysine polypeptide by a standalone adenylation domain involved in streptothricin biosynthesis. ${ }^{14}$ Dr Wendy L Kelly (Georgia Institute of Technology) described production of thiostrepton analogs and their structureactivity relationships. Thr7 of thiostrepton, shown to be crucial for its activity, should be responsible for contact with $23 \mathrm{~S}$ rRNA of the ribosome. ${ }^{15}$ Dr Hiroyasu Onaka (Toyama Prefectural University) reported a novel co-culture method utilizing the mycolic acidcontaining bacterium, Tsukamurella pulmonis TP-B0596. In the presence of this bacterium, S. lividans TK23 produces pigments in good yield. ${ }^{16}$ Professor Eric W Schmidt (University of Utah) presented the discovery of biosynthetic gene clusters for producing nocapyrone, patellins and patellazole $\mathrm{C}$ from the symbiotic bacteria in marine animals. To find the patellazole biosynthetic gene cluster from uncultured bacteria, the whole genome of the host and symbiont were sequenced. ${ }^{17}$ Engineering the patellin biosynthetic pathway in E. coli to produce novel peptide molecules was also discussed. ${ }^{18}$ Dr Toshiyuki Wakimoto (University of Tokyo) described identification of the calyculin biosynthetic gene cluster from the marine sponge Discodermia calyx. This group used PCR with degenerate primers targeting ketosynthase (KS) of acyltransferaseless PKS to produce probes, which were used to screen a metagenomic library of the sponge. Dr Tsutomu Sato (Niigata University) presented characteri-

zation of a novel terpene cyclase from Bacillus megaterium, which accepts both tetraprenyl- $\beta$-curcumene (C35) and squalene (C30) as substrates. ${ }^{19}$ Dr Atsushi Minami (Hokkaido University) reported the reaction mechanism of C-methyltransferase of prenyl diphosphate in longestin biosynthesis based on its crystal structure. Dr Tomohisa Kuzuyama (University of Tokyo) described the reaction mechanism of CotB2, which is a diterpene cyclase from Streptomyces and is responsible for cyclooctatin production ${ }^{20}$ based on its crystal structure. The unusual rearrangement of carbon atoms of cyclooctatin was demonstrated using the ${ }^{13} \mathrm{C}$-labeled substrate and NMR techniques.

\section{NOVEL TRANSFORMATIONS AND ISOPRENOIDS}

Session 5 (Novel transformations and isoprenoids) opened with a lecture by Professor Tohru Dairi (Hokkaido University), who presented several reactions to produce fusicoccin in the fungus Phomopsis amygdali, and eliminated a hydroxyl group at the 12position of fusicoccin by gene disruption, resulting in production of compounds related to cotylenin A, which exhibits anticancer activity. ${ }^{21}$ Dr Hisashi Hemmi (Nagoya University) reported reactions of several enzymes, including G-1-P dehydrogenase, GGPP synthase, GGGP synthase and DGGGP synthase, which are essential for production of phospholipid in Archaea. ${ }^{22}$ Professor Shigehiko Kanaya (Nara Institute of Science and Technology) described construction of a species-metabolite and metabolite-enzyme database. Professor Greg Challis (University of Warwick, Coventry) discussed two unprecedented $\mathrm{C}-\mathrm{H}$ functionalization reactions including $\mathrm{C}-\mathrm{C}$ bond-forming reactions by novel Rieske nonheme iron-dependent oxygenases in streptorubin B and metacycloprodigiosin biosyntheses, as well as regiospecific nitration of L-tryptophan in thaxtomin biosynthesis. ${ }^{23}$

\section{GENOMICS, METABOLOMICS AND FUNCTIONAL GENOMICS}

Session 6 (Genomics, metabolomics and functional genomics) opened with a lecture by Professor Fumihiko Sato (Kyoto University), who reported fermentative production of $(S)$-reticuline, a key intermediate of benzylisoquinoline alkaloids, in E. coli grown in a simple medium without additional substrates. ${ }^{24}$ Professor Toni M Kutchan (Donald Danforth Plant Science Center) described transcriptome and metabolome analyses of Papaveraceae for investigation of benzylisoquinoline alkaloid biosynthesis. Dr Tomoyoshi Akashi (Nihon University) presented the characterization of a 2-hydroxyisoflavanone synthase in nonleguminous plant and a prenyltransferase responsible for glyceollin and other phytoalexin 
biosynthesis in soybean. ${ }^{25,26}$ Dr Hikaru Seki (Osaka University) reported activities of several P-450 enzymes responsible for sitespecific oxidations of $\beta$-amyrin. ${ }^{27}$ These oxidations were characterized by heterologous expression in the yeast. Dr Masaharu Mizutani (Kobe University) presented RNAi knockdown methods of P-450 enzymes involved in biosynthesis of steroidal saponin in plants, which oxidizes $\beta$-amyrin. Professor Joe Chappell (University of Kentucky) described recent progresses of transcriptome and metabolome analyses of medical plants. The biosynthesis of a sesquiterpene, valerena-1(9), 10-diene was discussed as a representative of the progress made. Professor Kazuki Saito (Chiba University) reviewed an integrated transcriptomics and metabolomics strategy, and presented an application of this strategy to plants to produce camptothecin or quinolizidine alkaloids. ${ }^{28}$ Both metabolomics and transcriptomics have also been shown to be profitable approaches in the study of plant biosynthetic pathways.

\section{NOVEL TRANSFORMATIONS, EXPRESSION, DRUG DISCOVERY AND TOOLS}

Session 7 (Novel transformations, expression, drug discovery and tools) opened with a lecture by Professor Bradley S Moore (University of California, San Diego), who presented coupling reactions catalyzed by halogenases and haloperoxidases in the biosyntheses of merochlorin C, marinopyrrole A and chlorizidine A. ${ }^{29}$ The regio- and stereospecificities of these enzymes were clarified in this lecture. Professor Haruo Ikeda (Kitasato University) reported construction of mega-deletion mutants of $S$. avermitilis. These mutants were shown to be very useful for expressing biosynthetic clusters of several antibiotics, including streptomycin and cephamycin. ${ }^{30}$ Dr Shunji Takahashi (RIKEN) described screening and identification of $\beta$-carboline compound (NPD2639) from a chemical library (Riken NPDepo) as a biomediator that significantly increases the yields of secondary metabolites in Streptomyces. NPD2639 was shown to bind the LuxR type regulator in the reveromycin A biosynthetic gene cluster. ${ }^{31}$ Professor Rolf Müller (Saarland University) reported several unique biosynthetic pathways found in myxobacteria, including elansolids and aurachin biosyntheses. Detection of the compounds from myxobacteria was performed in a high-throughput manner using LC-MS. Construction of the heterologous expression system utilizing linear-linear homologous recombination of several gene clusters was also discussed. ${ }^{32}$ Professor Jun Ishikawa (National Institute of Infectious Diseases) presented the construction of a web-based support tool (2nd Find) to find secondary metabolite biosynthetic genes on the basis of Pfam domains. Dr Masaki Fujita (Hokkaido University) described the finding of a bisucaberinproducing clone from the metagenomic library constructed from deep sea samples, ${ }^{33}$ and the switching of bisucaberin production to desferrioxamine $\mathrm{E}$ production by exchanging an enzyme catalyzing macrocyclization. Dr Fumitaka Kudo (Tokyo Institute of Technology) discussed the reactions of biosynthetic enzymes to produce aminoglycoside antibiotics, with particular emphasis on the unique deamination process to generate a hydroxyl group at the $\mathrm{C} 2{ }^{\prime}$-position of kanamycin, which was catalyzed by both KanJ ( $\alpha$-ketoglutaratedependent nonheme iron dioxygenase) and KanK (NADPHdependent reductase). ${ }^{34}$ Professor Yasuo Ohnishi (University of Tokyo) reported a series of reactions to produce ferroverdin A. The in vitro reactions of nitroso-forming oxidization of 3-amino4-hydroxybenozic acid as well as esterification via AMP ester of 4-hydroxy-3-nitrosobenzamide involved in this pathway were discussed in detail. Professor Hung-wen Liu (University of Texas, Austin) described the biosynthetic pathway of BE-7585A, a thiosugar-containing angucycline. ${ }^{35}$ The introduction of a sulfate group into the glucose scaffold by biosynthetic enzymes was discussed in detail.

\section{NOVEL TRANSFORMATIONS, GENOME MINING, DRUG DISCOVERY AND TOOLS}

Session 8 (Novel transformations, genome mining, drug discovery and tools) opened with a lecture by Professor Tadhg Begley (Texas A\&M University), who presented the biosynthetic pathways to the thiazole and pyrimidine heterocycles of thiamin in S. cerevisiae. A thiazole sulfur was shown to be derived from Cys205 of the thiazole synthase (THI4) and the N3-C4-NH2 fragment of the pyrimidine is derived from His66 of the pyrimidine synthase (THI5). ${ }^{36}$ Professor Koji Ichinose (Musashino University) reported the oxidations involved in the production of actinorhodin and its related compounds by a two-component oxidase-reductase system. Dr Taifo Mahmud (Oregon State University) discussed the diverse reactions catalyzed by sedoheptulose 7-phosphate cyclases. The genome mining and biochemical studies revealed the novel sedoheptulose 7-phosphate cyclase from Actinosynnema mirum to yield 2-epi-valiolone. ${ }^{37}$ Professor Jörn Piel (University of Bonn) reported the biosynthetic gene cluster of polytheonamide, a giant ribosomal peptide, isolated from bacterial symbionts of the marine sponge. An unusual radical-SAM enzyme was shown to be involved in amino acid epimerization. Professor Tadashi Eguchi (Tokyo Institute of Technology) described biosynthesis of a macrolactam antibiotic, vicenistatin, in which $(2 S, 3 S)$-3-methylaspartate was used as a starter unit of PKS and the terminal acyl group of the elongated intermediate was cleaved by the peptidase VinJ before macrolactam formation. ${ }^{38}$

\section{CLOSING SESSION}

Session 9 (Closing session) opened with a lecture by Professor Craig A Townsend (Johns Hopkins University) who presented the detailed investigation of the reaction catalyzed by catalytic domains, such as KS, malonyl-CoA: acyl carrier protein (ACP) transacylase (MAT), starter unit: ACP transacylase (SAT), product template and thioesterase/Claisen cyclase (TE/CLC) domains in PksA, the norsolorinic acid anthrone-producing PKS. The conformation formed by SAT-KS-MAT and ACP domains was important for priming of hexanoyl groups and the number of condensation reactions of malonyl-CoAs to yield hexanoyl-primed octaketides. ${ }^{39}$ In addition, TE/CLC was shown to have an editing role to cleave the thioesters of acetyl-ACP and hexanoyl-ACP, and decrease the amounts of derailment products derived from triketide intermediate. ${ }^{39}$ Professor Hiroyuki Osada (RIKEN) presented the biosynthesis of reveromycin A (RM-A) and genetic engineering to produce various RM-A derivatives. In vitro experiments showed that the post-PKS biosynthetic intermediates RM-Ala was converted to a dihydroketone intermediate by RevG, and the stereochemistry of the intermediate was controlled by RevJ, resulting in RM-A3a, which has a spiroacetal ring and shows $15 \mathrm{~S}$ stereochemistry. ${ }^{31}$ Furthermore, two enzymes-RevH (flavin monooxygenase) and RevN (esterase)—catalyzed the Baeyer-Villiger oxidation and cleavage of the $\mathrm{C} 3$ unit derived from propionate from the precursor.

There were many discussions among the participants in the poster sessions and evening socials during the conference. A walking tour to Himeji Castle was arranged in the afternoon of the fourth day of the conference.

Recent progress in DNA sequencing technology has enabled us to easily find genes encoding biosynthetic enzymes, thus accelerating the biosynthetic study of bacterial and fungal metabolites. Transcriptome 
analyses of plants will facilitate the further elucidation of plant biosynthetic pathways with the help of metabolomics. Several secondary metabolite biosynthetic gene clusters from symbiotic bacteria in marine animals were reported at this meeting. These bacteria seem to be unexploited sources for such biosynthetic genes. Advances in heterologous expression systems are required for their utilization, because they often consist of numerous genes. In this meeting, many crystallographic studies of enzymes were reported. Understanding the reaction mechanisms of the enzymes from these studies would be very helpful for the exploitation of novel enzyme reactions, which would pave the way for expanding the diversity of natural products. The 9th US-Japan Seminar on the Biosynthesis of Natural Products is planned to be held in the USA in 4 years.

\section{ACKNOWLEDGEMENTS}

The conference was supported by a grant from the Ministry of Education, Culture, Sports, Science and Technology of Japan, the Hyogo International Association, the Uehara Memorial Foundation, the Naito Foundation, the Research Foundation for Pharmaceutical Sciences, the Nakauchi Foundation, the International Exchange Activities of the Faculty of Agriculture, the University of Tokyo and Suntory Foundation for Life Sciences.

1 Fujii, R. et al. Total biosynthesis of diterpene aphidicolin, a specific inhibitor of DNA polymerase $\alpha$ : heterologous expression of four biosynthetic genes in Aspergillus oryzae. Biosci. Biotechnol. Biochem. 75, 1813-1817 (2011).

2 Hotta, K. et al. Enzymatic catalysis of anti-Baldwin ring closure in polyether biosynthesis. Nature 483, 355-358 (2012).

3 Valenzano, C. R., Lawson, R. J., Chen, A. Y., Khosla, C. \& Cane, D. E. The biochemical basis for stereochemical control in polyketide biosynthesis. J. Am. Chem. Soc. 131, 18501-18511 (2010).

4 Horsman, G. P., Chen, Y., Thorson, J. S. \& Shen, B. Polyketide synthase chemistry does not direct biosynthetic divergence between 9- and 10-membered enediynes. Proc. Nat/ Acad. Sci. USA 107, 11331-11335 (2010).

5 Morita, H. et al. Synthesis of unnatural alkaloid scaffolds by exploiting plant polyketide synthase. Proc. Natl Acad. Sci. USA 108, 13504-13509 (2011).

6 Piasecki, S. K. et al. Employing modular polyketide synthase ketoreductases as biocatalysts in the preparative chemoenzymatic syntheses of diketide chiral building blocks. Chem. Biol. 18, 1331-1340 (2012).

7 Morita, H. et al. Structural basis for the one-pot formation of the diarylheptanoid scaffold by curcuminoid synthase from Oryza sativa. Proc. Natl Acad. Sci. USA 107 19778-19783 (2010).

8 Crawford, J. M. et al. Structural basis for biosynthetic programming of fungal aromatic polyketide cyclization. Nature 461, 1139-1143 (2009).

9 Ames, B. D. et al. Crystal structure and functional analysis of tetracenomycin ARO/ CYC: implications for cyclization specificity of aromatic polyketides. Proc. Natl Acad. Sci. USA 105, 5349-5354 (2008).

10 Arakawa, K., Tsuda, N., Taniguchi, A. \& Kinashi, H. The butenolide signaling molecules SRB1 and SRB2 induce lankacidin and lankamycin production in Streptomyces rochei. ChemBioChem 13, 1447-1457 (2012).

11 Kharel, M. K. \& Rohr, J. Delineation of gilvocarcin, jadomycin, and landomycin pathways through combinatorial biosynthetic enzymology. Curr. Opin. Chem. Biol. 16, 150-161 (2012).

12 Seshime, Y., Juvvadi, P. R., Kitamoto, K., Ebizuka, Y. \& Fujii, I. Identification of csypyrone B1 as the novel product of Aspergillus oryzae type III polyketide synthase CsyB. Bioorg. Med. Chem. Lett. 20, 4785-4788 (2010).

13 Davison, J. et al. Genetic, molecular, and biochemical basis of fungal tropolone biosynthesis. Proc. Natl Acad. Sci. USA 109, 7642-7647 (2012).

14 Maruyama, C. et al. A stand-alone adenylation domain forms amide bonds in streptothricin biosynthesis. Nat. Chem. Biol. 8, 791-797 (2012).
$15 \mathrm{Li}$, C., Zhang, F. \& Kelly, W. L. Mutagenesis of the thiostrepton precursor peptide at Thr7 impacts both biosynthesis and function. Chem. Commun. (Camb.) 48, 558-560 (2012).

16 Onaka, H., Mori, Y., Igarashi, Y. \& Furumai, T. Mycolic acid-containing bacteria induce natural-product biosynthesis in Streptomyces species. Appl. Environ. Microbiol. 77, 400-406 (2011)

17 Donia, M. S. et al. Complex microbiome underlying secondary and primary metabolism in the tunicate-Prochloron symbiosis. Proc. Natl Acad. Sci. USA 108, 1423-1432 (2012).

18 Tianero, M. D., Donia, M. S., Young, T. S., Schultz, P. G. \& Schmidt, E. W. Ribosomal route to small-molecule diversity. J. Am. Chem. Soc. 134, 418-425 (2012).

19 Sato, T., Hoshino, H., Yoshida, S., Nakajima, M. \& Hoshino, T. Bifunctional triterpene/ sesquarterpene cyclase: tetraprenyl- $\beta$-curcumene cyclase is also squalene cyclase in Bacillus megaterium. J. Am. Chem. Soc. 133, 17540-17543 (2012).

$20 \mathrm{Kim}, \mathrm{S}$. Y. et al. Cloning and heterologous expression of the cyclooctatin biosynthetic gene cluster afford a diterpene cyclase and two P450 hydroxylases. Chem. Biol. 16, 736-743 (2009)

21 Ono, Y. et al. Dioxygenases, key enzymes to determine the aglycon structures of fusicoccin and brassicicene, diterpene compounds produced by fungi. J. Am. Chem. Soc. 133, 2548-2555 (2011)

22 Yokoi, T., Isobe, K., Yoshimura, T. \& Hemmi, H. Archaeal phospholipid biosynthetic pathway reconstructed in Escherichia coli. Archaea 2012, 1-9, (2012).

23 Sydor, P. K. et al. Regio- and stereodivergent antibiotic oxidative carbocyclizations catalysed by Rieske oxygenase-like enzymes. Nat. Chem. 3, 388-392 (2011).

24 Nakagawa, A. et al. A bacterial platform for fermentative production of plant alkaloids. Nat. Commun 2, 1-8 (2011).

25 Akashi, T., Aoki, T. \& Ayabe, S. Molecular and biochemical characterization of 2-hydroxyisoflavanone dehydratase. Involvement of carboxylesterase-like proteins in leguminous isoflavone biosynthesis. Plant Physiol 137, 882-891 (2005).

26 Akashi, T., Sasaki, K., Aoki, T., Ayabe, S. \& Yazaki, K. Molecular cloning and characterization of a cDNA for pterocarpan 4-dimethylallyltransferase catalyzing the key prenylation step in the biosynthesis of glyceollin, a soybean phytoalexin. Plant Physiol 149, 683-693 (2009).

27 Fukushima, E. O. et al. CYP716A subfamily members are multifunctional oxidases in triterpenoid biosynthesis. Plant Cell. Physiol 52, 2050-2061 (2012).

28 Asano, T. et al. Suppression of camptothecin biosynthetic genes results in metabolic modification of secondary products in hairy roots of Ophiorrhiza pumila. Phytochemistry (e-pub ahead of print 29 May 2012).

29 Kaysser, L. et al. Merochlorins A -D, cyclic meroterpenoid antibiotics biosynthesized in divergent pathways with vanadium-dependent chloroperoxidases. J. Am. Chem. Soc 134, 11988-11991 (2012).

30 Komatsu, M., Uchiyama, T., Omura, S., Cane, D. E. \& Ikeda, H. Genome-minimized Streptomyces host for the heterologous expression of secondary metabolism. Proc. Natl Acad. Sci. USA 107, 2646-2451 (2010).

31 Takahashi, S. et al. Reveromycin A biosynthesis uses RevG and RevJ for stereospecific spiroacetal formation. Nat. Chem. Biol. 7, 461-468 (2011).

$32 \mathrm{Fu}$, J. et al. Full-length RecE enhances linear-linear homologous recombination and facilitates direct cloning for bioprospecting. Nat. Biotechnol. 30, 440-446 (2012).

33 Fujita, M. J., Kimura, N., Yokose, H. \& Otsuka, M. Heterologous production of bisucaberin using a biosynthetic gene cluster cloned from a deep sea metagenome. Mol. Biosyst. 8, 482-485 (2012).

34 Sucipto, H., Kudo, F. \& Eguchi, T. The last step of kanamycin biosynthesis: unique deamination reaction catalyzed by the $\alpha$-ketoglutarate-dependent nonheme Iron dioxygenase KanJ and the NADPH-dependent reductase KanK. Angew. Chem. Int Ed. Eng/ 51, 3428-3431 (2012).

35 Sasaki, E. \& Liu, H. W. Mechanistic studies of the biosynthesis of 2-thiosugar: evidence for the formation of an enzyme-bound 2-ketohexose intermediate in BexXcatalyzed reaction. J. Am. Chem. Soc. 132, 15544-15546 (2011).

36 Chatterjee, A. et al. Saccharomyces cerevisiae THI4p is a suicide thiamine thiazole synthase. Nature 478, 542-546 (2012).

37 Asamizu, S., Xie, P., Brumsted, C. J., Flatt, P. M. \& Mahmud, T. Evolutionary divergence of sedoheptulose 7-phosphate cyclases leads to several distinct cyclic products. J. Am. Chem. Soc. 134, 12219-12229 (2012).

38 Shinohara, Y., Kudo, F. \& Eguchi, T. A natural protecting group strategy to carry an amino Acid starter unit in the biosynthesis of macrolactam polyketide antibiotics. J. Am. Chem. Soc. 133, 18134-18137 (2011)

39 Vagstad, A. L., Bumpus, S. B., Belecki, K., Kelleher, N. L. \& Townsend, C. A Interrogation of global active site occupancy of a fungal iterative polyketide synthase reveals strategies for maintaining biosynthetic fidelity. J. Am. Chem. Soc. 134, 6865-6877 (2012) 